\title{
Comparative study of optical properties of the one-dimensional multilayer Period- Doubling and Thue-Morse quasi-periodic photonic crystals
}

\author{
Yassine Bouazzi ${ }^{1}$, Mounir Kanzari ${ }^{1}$ \\ ${ }^{1}$ Laboratoire de Photovoltaïque et Matériaux Semi-conducteurs (LPMS), Ecole Nationale d'Ingénieurs de Tunis, Université \\ de Tunis El Manar, Tunisie \\ *corresponding author, E-mail: yassine.bouazzi@gmail.com
}

\begin{abstract}
The last decades have witnessed the growing interest in the use of photonic crystal as a new material that can be used to control electromagnetic wave. Actually, not only the periodic structures but also the quasi-periodic systems have become significant structures of photonic crystals. This work deals with optical properties of dielectric ThueMorse multilayer and Period-Doubling multilayer generated by:

Thue-Morse: $\mathrm{H} \rightarrow \mathrm{HL}, \mathrm{L} \rightarrow \mathrm{LH}$

Period-Doubling: $\mathrm{H} \rightarrow \mathrm{HL}, \mathrm{L} \rightarrow \mathrm{HH}$

Where $\mathrm{H}$ and $\mathrm{L}$ are two elementary layers with refractive indices $\mathrm{nL}=1.45$ and $\mathrm{nH}=2.3$ respectively, and a thickness on the order of $\lambda / 4$ where $\lambda$ is the wavelength of the light. In the following numerical investigation, we chose $\mathrm{SiO} 2(\mathrm{~L})$ and $\mathrm{TiO} 2(\mathrm{H})$ as two elementary layers. We use the so-called Transfer Matrix Method (TMM) to determine the reflection spectra of the structures. Based on the representation of the transmittance spectra in the visible range a comparative analysis depending on the iteration number, number of layers and incidence angle is presented.
\end{abstract}

\section{Introduction}

The past two decades have seen a tremendous increase in interest in the field of photonic crystals [1-4]. These microstructured optical materials are characterized through a spatially periodic or quasiperiodic modulation in their index of refraction [5-7]. During the last decade we have come to realize that ordered matter domains can be suitably expanded to embrace not only periodic arrangements but quasiperiodic ones as well $[6,8]$.

The recent studies of PBG quasiperiodic systems, were directed towards the quasiperiodic multilayer structures, in particular the one-dimensional structures with distribution of Fibonacci, Cantor, Thue-Morse, PeriodDoubling..., in order to find best optical performances for the contribution in the optical devices development. One of the most important properties of a quasiperiodic photonic crystal is that it presents a gap which makes it possible to control the spontaneous emission [9] by prohibiting the propagation of the electromagnetic wave. We cite among other applications, optical fibers, waveguides operating at wavelengths of telecommunications, light emitting diodes.

This study focuses on onedimensional structures using the Transfer Matrix Method (TMM) for the calculation of the transmission coefficients or the reflection coefficients for the two types of polarizations: S-polarization (TE mode) and Ppolarization (TM mode).

\section{Model and calculation method}

\subsection{Thue-Morse sequence}

The Thue-Morse (TM) sequence is extensively studied in the mathematical literature as the prototype of a sequence generated by the substitution rule $\mathrm{H} \rightarrow \mathrm{HL}, \mathrm{L} \rightarrow \mathrm{LH} \quad[10-12]$, we can deduce all subsequent orders of the $\mathrm{S}_{\mathrm{k}}$ sequence, where $\mathrm{k}$ is the order.

One can formulate the sequence in the following way:

$$
\left\{\begin{array}{l}
\mathrm{S}_{1}=\mathrm{H} \\
\overline{\mathrm{S}_{1}}=\mathrm{L} \\
\mathrm{S}_{\mathrm{k}+1}=\mathrm{S}_{\mathrm{k}} \overline{\mathrm{S}_{\mathrm{k}}} \\
\overline{\mathrm{S}_{\mathrm{k}+1}}=\overline{\mathrm{S}_{\mathrm{k}}} \mathrm{S}_{\mathrm{k}}
\end{array}\right.
$$

An example of generation of the Thue-Morse sequence is presented below (Fig. 1):

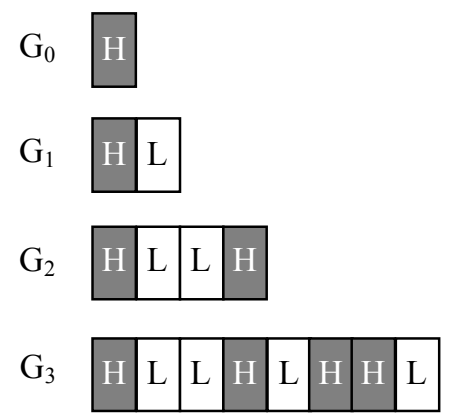

Figure 1: Generation of the Thue-Morse sequence 
The number of layers in this increases geometrically, $T M_{k}=2^{k} \forall k \in I N(\mathrm{x})$, where $k$ indicates the iteration order. The calculation by iteration can validate that the number of low $(\mathrm{L})$ and high $(\mathrm{H})$ index layers are equal respectively:

$\mathrm{TM}_{\mathrm{k}}^{\mathrm{L}}=2^{\mathrm{k}-1}$

and

$\mathrm{TM}_{\mathrm{k}}^{\mathrm{H}}=2^{\mathrm{k}-1}$

\subsection{Period-Doubling sequence}

The Period-Doubling substitution sequence can be defined via a binary alphabet $\{\mathrm{H}, \mathrm{L}\}$ and a set of simple mapping rules $\mathrm{H} \rightarrow \mathrm{HL}, \mathrm{L} \rightarrow \mathrm{HH}$, with the symbol $\mathrm{H}$ as an initiator. The first several PD generations are $\mathrm{H}, \mathrm{HL}$, HLHH, HLHHHLHL, etc $[10,13,14]$. A schematic presentation of PD multilayer is shown in Fig. 2.

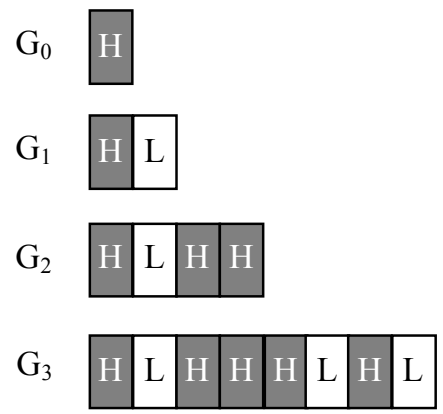

Figure 2: Generation of the Period-Doubling sequence

Otherwise, the Period-Doubling, $\mathrm{PD}_{\mathrm{j}}$ with $\mathrm{j}=0,1,2$ ..., are characterized by the inflation scheme:

$\left\{\begin{array}{l}S_{0}=H \\ S_{1}=H L \\ S_{j+1}=S_{j} S_{j-1}^{2}\end{array}\right.$

For such a structure the number of the layers, high index $(\mathrm{H})$ and low index $(\mathrm{L})$, at the $\mathrm{i}^{\text {th }}$ iteration is given by applying the initial conditions: $\mathrm{PD}_{1}=1$ and $\mathrm{PD}_{2}=2$, we obtain then, is equal to:

- The total layer number (high and low index): $P D_{i}=2^{i} \forall i \in I N$

- The low index layers number (by applying the initial conditions: $\mathrm{PD}_{1}=1$ and $\mathrm{PD}_{2}=0$ ):

$$
\mathrm{PD}_{\mathrm{i}}^{\mathrm{L}}=\frac{2}{3} 2^{\mathrm{i}}+\frac{1}{3}(-1)^{\mathrm{i}} \quad \forall \mathrm{i} \geq 2
$$

- The number of low index layers:

$$
\mathrm{PD}_{\mathrm{i}}^{\mathrm{H}}=\frac{1}{3} 2^{\mathrm{i}}-\frac{1}{3}(-1)^{\mathrm{i}} \quad \forall \mathrm{i} \geq 2
$$

\subsection{Transfer Matrix Method (TMM)}

We employ the Transfer Matrix Method (TMM), is used to extract transmission and reflection spectra, consider their sensitivity to material and geometrical variation. It can solve the problem of the photonic band structures and the scattering (transmission and reflection) spectra. For stratified layers within m layers (Fig. 3), the amplitudes of the electric fields of incident wave $\mathrm{E}_{0}^{+}$, reflected wave $\mathrm{E}_{0}^{-}$ and transmitted wave $\mathrm{E}_{\mathrm{m}+1}^{+}$after $\mathrm{m}$ layers can be related via the following matrix [15]:

$$
\left(\begin{array}{c}
E_{0}^{+} \\
E_{0}^{-}
\end{array}\right)=\frac{C_{1} C_{2} C_{3} \ldots C_{m+1}}{t_{1} t_{2} t_{3} \ldots t_{m+1}}\left(\begin{array}{l}
E_{m+1}^{+} \\
E_{m+1}^{-}
\end{array}\right)
$$

The $C_{j}$ (propagation matrix) for the $j^{\text {th }}$ sequence can be written:

$$
\begin{aligned}
& \mathrm{C}_{\mathrm{j}}=\left(\begin{array}{cc}
\exp \left(\mathrm{i} \phi_{\mathrm{j}-1}\right) & \mathrm{r}_{\mathrm{j}} \exp \left(-\mathrm{i} \phi_{\mathrm{j}-1}\right) \\
\mathrm{r}_{\mathrm{j}} \exp \left(\mathrm{i} \phi_{\mathrm{j}-1}\right) & \exp \left(-\mathrm{i} \phi_{\mathrm{j}-1}\right)
\end{array}\right) \\
& \phi_{\mathrm{j}-1} \text { indicate the phase shift of the wave }
\end{aligned}
$$
between $(j-1)^{\text {th }}$ and $j^{\text {th }}$ boundaries and can be obtained by:

$\phi_{0}=0$

$\phi_{\mathrm{j}-1}=\frac{2 \pi}{\lambda} \hat{\mathrm{n}}_{\mathrm{j}-1} \mathrm{~d}_{\mathrm{j}-1} \cos \theta_{\mathrm{j}-1}$

The Fresnel coefficients $t_{j}$ and $r_{j}$ can be expressed as follows by using the complex refractive index $\hat{\mathrm{n}}_{\mathrm{j}}$ and the complex refractive angle $\theta_{\mathrm{j}}$.

For parallel P-polarization (TM mode):

$r_{j p}=\frac{\hat{n}_{j-1} \cos \theta_{j}-\hat{n}_{j} \cos \theta_{j-1}}{\hat{n}_{j-1} \cos \theta_{j}+\hat{n}_{j} \cos \theta_{j-1}}$

$$
\mathrm{t}_{\mathrm{jp}}=\frac{2 \hat{\mathrm{n}}_{\mathrm{j}-1} \cos \theta_{\mathrm{j}-1}}{\hat{\mathrm{n}}_{\mathrm{j}-1} \cos \theta_{\mathrm{j}}+\hat{\mathrm{n}}_{\mathrm{j}} \cos \theta_{\mathrm{j}-1}}
$$

Moreover, for perpendicular S- polarization (TE mode):

$$
r_{j s}=\frac{\hat{n}_{j-1} \cos \theta_{j-1}-\hat{n}_{j} \cos \theta_{j}}{\hat{n}_{j-1} \cos \theta_{j-1}+\hat{n}_{j} \cos \theta_{j}}
$$

$$
\mathrm{t}_{\mathrm{js}}=2 \frac{\hat{\mathrm{n}}_{\mathrm{j}-1} \cos \theta_{\mathrm{j}-1}}{\hat{\mathrm{n}}_{\mathrm{j}-1} \cos \theta_{\mathrm{j}-1}+\hat{\mathrm{n}}_{\mathrm{j}} \cos \theta_{\mathrm{j}}}
$$

For both polarizations $\mathrm{S}$ and $\mathrm{P}$ the transmittance energy $\mathrm{T}$ are reduced as: 


$$
\begin{aligned}
& \mathrm{T}_{\mathrm{S}}=\operatorname{Re}\left(\frac{\hat{\mathrm{n}}_{\mathrm{m}+1} \cos \theta_{\mathrm{m}+1}}{\hat{\mathrm{n}}_{0} \cos \theta_{0}}\right)\left|\mathrm{t}_{\mathrm{S}}\right|^{2} \\
& \mathrm{~T}_{\mathrm{P}}=\operatorname{Re}\left(\frac{\hat{\mathrm{n}}_{\mathrm{m}+1} \cos \theta_{\mathrm{m}+1}}{\hat{\mathrm{n}}_{0} \cos \theta_{0}}\right)\left|\mathrm{t}_{\mathrm{P}}\right|^{2}
\end{aligned}
$$

Re: indicates the real part.

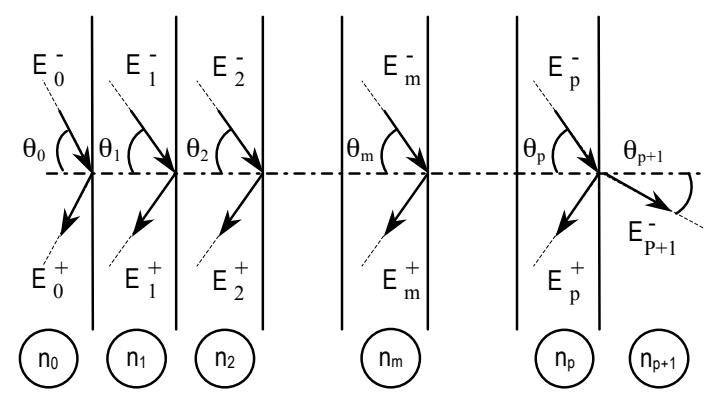

Figure 3: Multi-layer system with p components.

Basing on the matrix method we make an iterative algorithm calculation which enables us to determine the transmission spectra's of the study structures, we developed thereafter a script under the MATLAB computer programming language, which can extract the photometric response of a filter constructed by a dielectric multilayer system according to the distribution of Period-Doubling and Thue-Morse.

\section{Numerical results and discussion}

For the sake of numerical calculation we take $\mathrm{SiO}_{2} / \mathrm{TiO}_{2}$ dielectric material with low and high index contrast. The refractive index for is $\mathrm{SiO}_{2} \mathrm{n}_{\mathrm{L}}=1.45$ and for is $\mathrm{TiO}_{2}$ $\mathrm{n}_{\mathrm{H}}=2.3$, with central wavelength equal to $\lambda_{0}=0.5 \mu \mathrm{m}$.

We studied under normal incidence the effect of the iterations on the reflection spectra of the structures built according to the Period-doubling and Thue-Morse sequences (fig. 4), shows that when the number of iteration $(\mathrm{k})$ increases, the spectral bands narrow and become increasingly cumbersome, And then the appearance of new PBG in the visible spectral range.

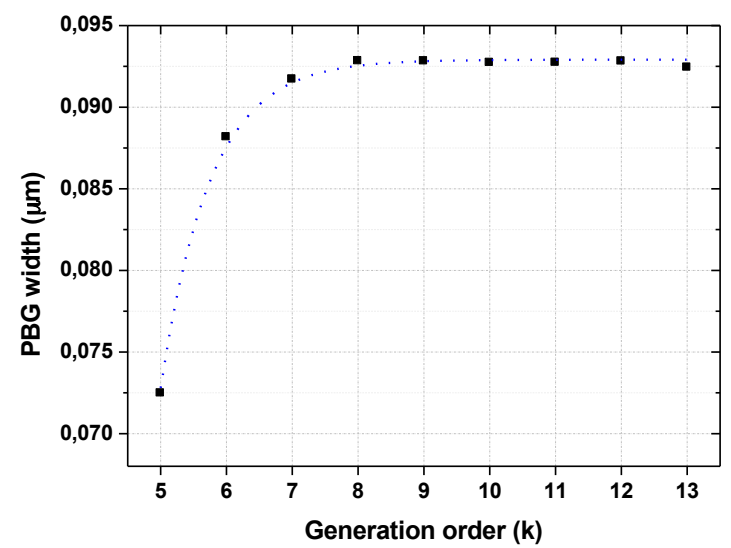

Figure 5: Evolution of BPG width (around $\lambda_{0}$ ) according to the generation order $(\mathrm{k})$ of the PeriodDoubling sequence

For a dielectric multi-layer system according to the PeriodDoubling distribution, the transmission spectra (Fig. 4) shows the presence of a photonic band gap centred around $\lambda_{0}=0.5 \mu \mathrm{m}$. The PBG width increases exponentially (Fig. 5) as a function of the iteration (k). It is also noticed that for large values of the generation order $(\mathrm{k})$ (starting from the $8^{\text {th }}$ iteration), we see the presence of multitudes of transmission peaks where the multilayer system become like a polychromatic filter.

On the other hand for a photonic system according to the distribution of Thue-Morse one notes the presence of the oscillating peaks around $\lambda_{0}$, where the value of transmission to $\lambda_{0}=0.5 \mu \mathrm{m}$ is null whatever the order of generation.

We show the appearance of optical windows (transmission peak) in the band gap for the photonic multilayer according to the Thue-Morse distribution, these optical windows always keeps a fixed position in the spectral range whatever the value of generation $(k \geq 5)$ of multilayer systems:

- 1 st optical window at $0.6172 \mu \mathrm{m}$

- 2nd optical window at $0.7034 \mu \mathrm{m}$ 

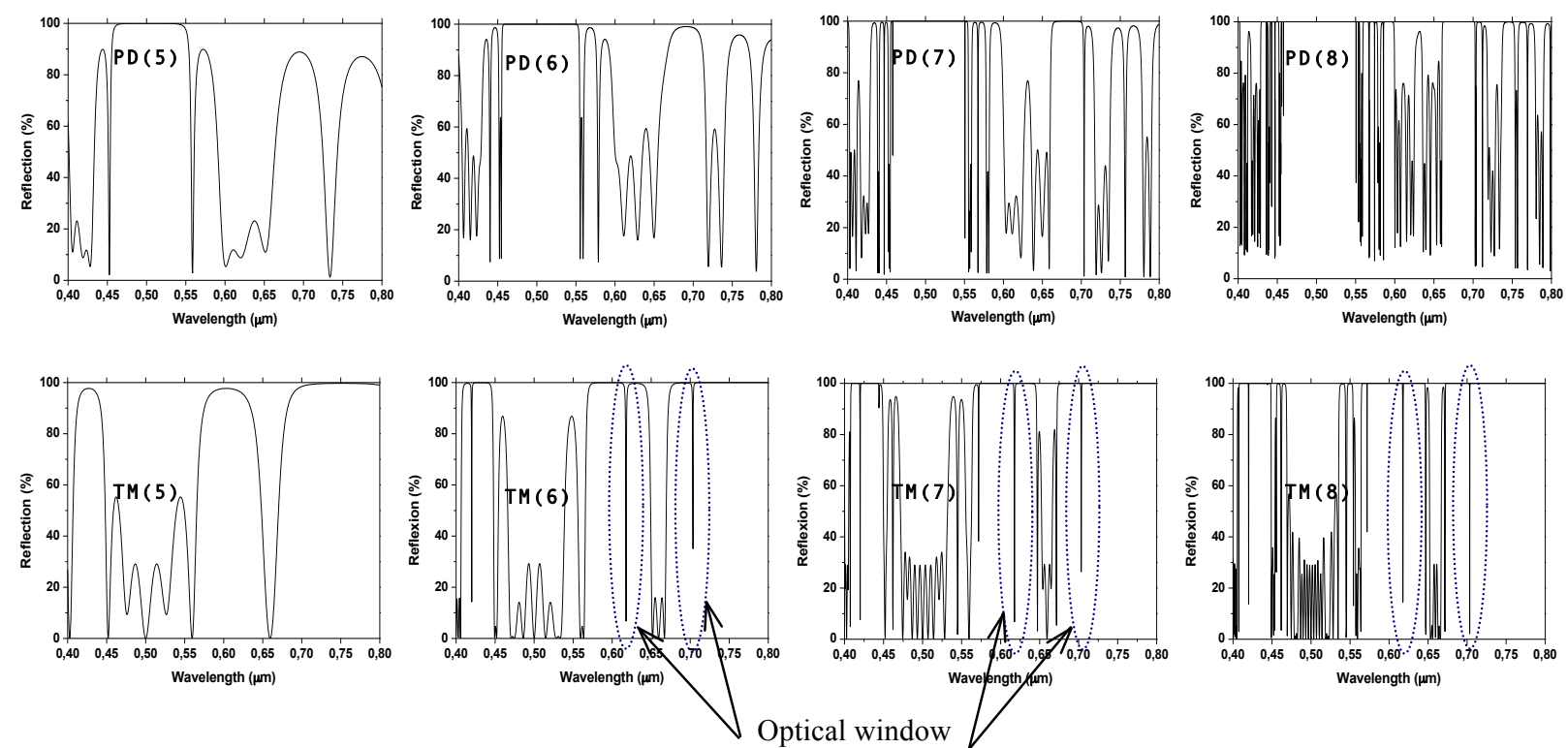

Figure 4: Reflection spectrum versus the multilayer structure according to the distribution of Period-Doubling and Thue-Morse distributions for the $5^{\text {th }}, 6^{\text {th }}, 7^{\text {th }}, 8^{\text {th }}$, generations.

To study the polarization effect we plotted the reflection spectra of multilayer systems according to the distributions of Period-Doubling and Thue-Morse, depending on incidence angle $\theta(\mathrm{rad})$ of the polarized wave in TE and TM modes (fig.6 and fig.7). And then projecting the spectrum on the (oxy) plane to find the band gap, the white areas represent propagation bands, and the blue areas forbidden bands, where $\mathrm{R}>99.9$ $\%$. And we chose the $5^{\text {th }}$ generation of the sequences of Thue-Morse TM (5) and Period-Doubling PD (5) (Fig.6 and Fig 7).

(a)

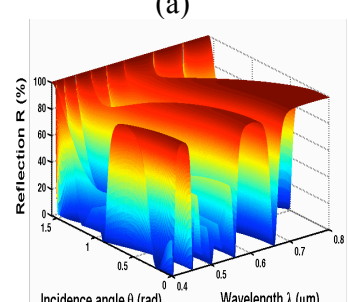

Incidence angle $\theta$ (rad) $)_{0.4}^{0.5}$ Wavelengh $\lambda(\mathrm{um})$

(c)

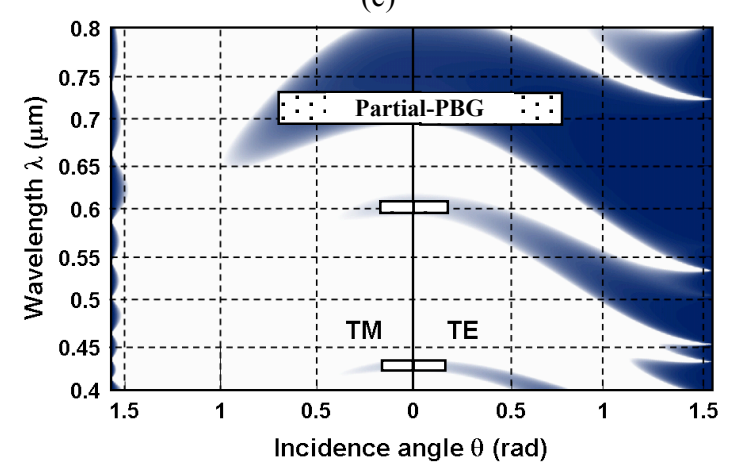

Figure 6: (a) Transmission spectra of the photonic multilayer system according to the distribution of Thue-Morse for the 5th generation TM(5), mode TM. (b) Transmission spectra of the photonic multilayer system according to the distribution of Thue-Morse for the 5th generation TM(5), mode TE. (c) Photonic band structures for the $\operatorname{TM}(5)$, where $\mathrm{R}>99.9 \%$.

(a)

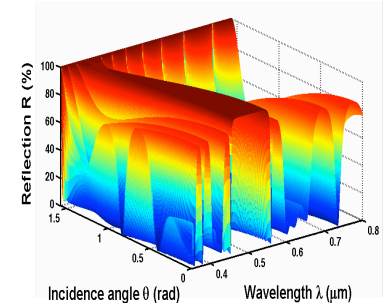

(b)

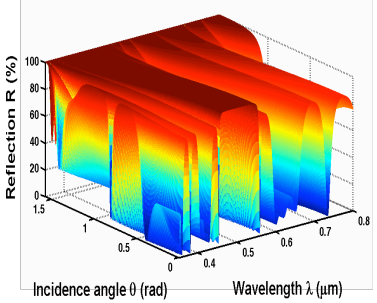

(c)

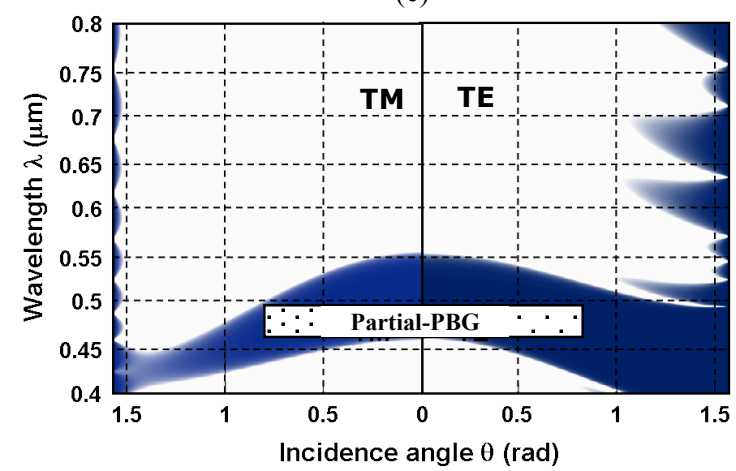

Figure 7: (a) Transmission spectra of the photonic multilayer system according to the distribution of Period-Doubling for the 5th generation PD(5), mode TM. (b) Transmission spectra of the 
photonic multilayer system according to the distribution of Period-Doubling for the 5th generation $\mathrm{PD}(5)$, mode TE. (c) Photonic band structures for the $\mathrm{PD}(5)$, where $\mathrm{R}>99.9 \%$.

As shown in Fig. 6.c and Fig. 7.c, the absence of unidirectional $\mathrm{PBG}$, but one notes the appearance of the partial forbidden band (partial-PBG), where the prohibition of propagation wave in the structure is limited in a defined interval of the opening angle of the incident wave.

The table 1 presents the structural and the optical aspects differences between the two photonic systems TM (5) and PD (5).

Table 1: Structural and optical aspects of the two photonic systems TM (5) and PD (5)

\begin{tabular}{|c|c|c|}
\hline & & \\
\hline & $\mathrm{TM}(5)$ & $\begin{aligned} \mathrm{PD}(5) \\
\end{aligned}$ \\
\hline Layer distribution & HLLHLHHLLHHLHLLHLHHLHLLHHLLHLHHL & HLHHHLHLHLHHHLHHHLHHHLHLHLHHHLHL \\
\hline Layer number & 32 & 32 \\
\hline $\begin{array}{l}\text { Omni-directional } \\
\text { PBG number }\end{array}$ & 0 & 0 \\
\hline $\begin{array}{c}\begin{array}{c}\text { Partial PBG } \\
\text { number }\end{array} \\
\end{array}$ & 3 & 1 \\
\hline \multirow{6}{*}{$\begin{array}{l}\text { Partial PBG } \\
\text { spectral range }\end{array}$} & $\begin{array}{l}\lambda \in[0.695 \mu \mathrm{m} 0.735 \mu \mathrm{m}] \\
\Delta \lambda=0.04 \mu \mathrm{m} \\
\text { with } \theta \in\left[\begin{array}{ll}0 & 0.226 \pi\end{array}\right]\end{array}$ & \multirow{6}{*}{$\begin{array}{l}\lambda \in[0.4628 \mu \mathrm{m} 0.4975 \mu \mathrm{m}] \\
\Delta \lambda=0.0347 \mu \mathrm{m} \\
\text { with } \theta \in\left[\begin{array}{ll}0 & 0.2629 \pi\end{array}\right]\end{array}$} \\
\hline & $\lambda \in[0.5946 \mu \mathrm{m} 0.6118 \mu \mathrm{m}]$ & \\
\hline & $\begin{array}{l}\Delta \lambda=0.0172 \mu \mathrm{m} \\
\text { with } \theta \in[00.0568 \pi]\end{array}$ & \\
\hline & $\lambda \in[0.4210 \mu \mathrm{m} 0.4355 \mu \mathrm{m}]$ & \\
\hline & $\Delta \lambda=0.0145 \mu \mathrm{m}$ & \\
\hline & with $\theta \in\left[\begin{array}{ll}0 & 0.0568 \pi\end{array}\right]$ & \\
\hline
\end{tabular}

\section{Conclusion}

The results presented in this paper are based on numerical calculations optical properties of the one-dimensional multilayer Period-Doubling and Thue-Morse quasiperiodic photonic crystals by using the Transfer Matrix Method. We show that for the normal incidence a photonic system according to the distribution of Period-Doubling, presents forbidden bands, the largest is centered around $\lambda_{0}=0.5 \mu \mathrm{m}$, where its width increases with the value of the iteration (k). On the other hand for the distribution of Thue-Morse one notes the absence of band gaps around $\lambda_{0}=0.5 \mu \mathrm{m}$, but notices the presence of the optical windows which appear in the other band gaps.

For oblique incidence we studied the transmission spectra for TE and TM mode, we found the absence of omnidirectional-PBG and the appearance of partial-PBG, where their numbers and their spectral ranges depend on type of distribution of multilayer.

\section{References}

[1] K. Busch, Photonic band structure theory: assessment and perspectives, Comptes Rendus Physique, Vol 3, Issue 1, pp 53-66, 2002

[2] B. Suthar, A. Bhargav, Tunable multi-channel filtring using 1-D photonic quantium well structure, Progress In Electromagnetics Research Letters, Vol. 27, pp 43-51, 2011.

[3] E. YABLONOVITCH, Inhibited Spontaneous Emission in Solid-State Physics and Electronics, Physical Review Letter, Vol. 58, N²0, 1987.
[4] E. YABLONOVITCH, Photonic Ban-Gap structure, J. Opt. Society, Vol 10, 1993

[5] A. Bahabad, R. Lifshitz, N. Voloch, A. Arie, Nonlinear photonic quasicrystals for novel optical devices, Phil. Mag. Vol 88, pp 2285-2293, 2008.

[6] E. Macia, Exploiting aperiodic designs in nanophotonic devices, Rep. Prog. Phys. Vol 75, Issue 3, pp. 036502, 2012.

[7] E. Macia, The role of aperiodic order in science and technology, Rep. Prog. Phys., Vol 69, pp 397-441, 2006.

[8] K. Buscha., G. von Freymannb, S. Lindenb, S.F. Mingaleeva,c, L. Tkeshelashvilia, M. Wegenerd, Periodic nanostructures for photonics, Physics Reports, Vol 444, pp 101-202, 2007.

[9] E. Ozbay, I. Bulu, K. Aydin, H. Caglayan, K. Guven, Physics and applications of photonic crystals, Photonics and Nanostructures - Fundamentals and Applications, Vol 2, pp 87-95, 2004.

[10] L. Kroon, R. Riklund, Renormalization of aperiodic model lattices: spectral properties, J. Phys. A: Math. Gen., Vol 36, pp 4519-4532, 2003.

[11] A.N. Poddubny, E. L. Ivchenko, Photonic quasicrystalline and aperiodic structures, Physica E, Vol 42, pp 1871-1895, 2010.

[12] G. Zhang, X.Yang, Y. Li, H. Song, Optical transmission through multi-component generalized Thue-Morse superlattices, Physica B,, Vol 405, pp 3605-3610, 2010.

[13] X. Wang, U. Grimm, M. Schreiber, Trace and antitrace maps for aperiodic sequences: Extensions and applications, Vol 62, Num 21, pp 14 020-14 031, 2000.

[14] V. Agarwal, Miguel E. Mora-Ramos, B. Alvarado-Tenorio, Optical properties of multilayered Period-Doubling and Rudin-Shapiro porous silicon dielectric heterostructures, Photonics and Nanostructures - Fundamentals and Applications, Vol 7, pp 63-68, 2009.

[15] K. Ben Abdelaziz, J. Zaghdoudi, M. Kanzari, B. Rezig, A broad omnidirectional reflection band obtained from 
deformed Fibonacci quasi-periodic one dimentional photonic crystals, J. Opt A : Pure Appl. Opt., Vol 7, pp 544-

549, 2005. 